\title{
Circadian regulation of auditory function
}

2

3 Vasiliki Basinou ${ }^{1}$, Jung-sub Park ${ }^{1,2}$, Christopher R. Cederroth ${ }^{1}$ and Barbara Canlon ${ }^{1}$, 4

5

6

\section{Author Affiliations}

1. Department of Physiology and Pharmacology Laboratory of Experimental Audiology Karolinska Institutet 17177 Stockholm, Sweden

\section{Department of Otolaryngology} Ajou University School of Medicine, 164 Worldcup-ro, Yeongtong-gu, Suwon 16499, Korea

Address correspondence to B. Canlon

Department of Physiology and Pharmacology

Laboratory of Experimental Audiology

Von Eulers väg 8

Karolinska Institutet

17177 Stockholm, SwedenTelephone: +46(8)524.87248; facsimile: +46(8)327026;

E-mail: Barbara.Canlon@ki.se 
ABR: Auditory brainstem response

ASR: Acoustic startle response

BDNF: Brain derived neurotrophic factor

CCG: Clock controlled genes

Clock: Circadian locomotor output cycle kaput

CRH: corticotropin releasing hormone

Cry: Cryptochrome

CT: Circadian time

DHF: 7, 8 dihydroxyflavone

NIHL: Noise-induced hearing loss

Per: Period

PTS: Permanent threshold shift

ROR: Retinoid-related orphan receptors

RORE: ROR response element

SCN: Suprachiasmatic nucleus

SPL: Sound pressure level

TTS: Temporal threshold shift

ZT: Zeitgeber time 


\section{Acknowledgments}

50

51

52

53

54

55

56

57

58

59

60

61

62

This work was supported by the Office of the Assistant Secretary of Defense for Health Affairs, through the Neurosensory and Rehabilitation under Award No. W81XWH-16-10032. Opinions, interpretations, conclusions and recommendations are those of the author and are not necessarily endorsed by the Department of Defense. Additional funds were obtained from the Communication Disorders of the National Institutes of Health R21DC013172 (B.C.), the Swedish Medical Research Council K2014-99X-22478-01-3 (B.C.), the National Institute on Deafness and other National Research Foundation of Korea grant (2013R1A2A2A01008325 and 2014R1A6A3A03058661) funded by the Ministry of Education of the Korean Government (J.S.P.), Karolinska Institutet (B.C., C.R.C.), Tysta Skolan (B.C., J.S.P.), Hörselforskningsfonden (B.C., G.L.), Lars Hiertas Minne (C.R.C.), Magnus Bergvalls (C.R.C.), Loo och Hans Ostermans (C.R.C.). We thank Min-jung Kim for help with the figures. The authors declare no competing financial interests. 


\section{Introduction: Circadian clocks}

Biological clocks evolved under the influence of rhythmic environmental cues, in order to provide an internal representation of time and allow organisms to exploit temporal niches (light/dark, cold/warm, wet/dry, etc.) with all the subsequent consequences. The environmental factors that influence the function of these clocks are called zeitgebers (German for "time-giver"). Among these factors are the light-dark cycle, temperature, feeding and social interactions. The light-dark cycle is considered a major zeitgeber and probably the most significant in evolution because it is an explicit predictor of the daily cycle, as well as the seasonal cycle (length of day fluctuations). However, the biological clock being an endogenous timing system is capable of generating biological rhythms even in the absence of environmental cues. This ability ensures that the physiological functions of an organism will continue even in temporal isolation.

When zeitgebers are not present, the biological clock sustains a rhythm of about $24 \mathrm{~h}$ which is called circadian rhythm (from the Latin words "circa" and "diem", meaning approximately one day), with a corresponding circadian time (CT). In order to produce an accurate $24 \mathrm{~h}$ period, the clock adjusts its rhythm on a daily basis. This adjustment is mediated mainly through entrainment to the daily light-dark cycle, meaning synchronization of the circadian time to the external time (Box 1). Consequently, the clock time relies on the rhythm of the zeitgeber and is referred as a zeitgeber time (ZT).

\subsection{The molecular clock: How clocks tell time}

Circadian rhythms are innate and are governed by genetically programmed mechanisms. The discovery of genes encoding circadian behavioral rhythms in Drosophila melanogaster (Konopka et al., 1971) initiated an intense scientific effort to identify genes that regulate the clock machinery, known as clock genes, and led to the hypothesis that rhythms are generated at the molecular level. An autoregulatory mechanism was proposed where clock gene mRNA is translated into protein, which then translocates into the nucleus and suppresses its own transcription (Hardin et al., 1990). Reduced levels of mRNA will then lead to reduced protein and eventually increased transcription, thereby restarting the cycle. Although simplified, this model represents the basic principle of the molecular clock. 
In mammals, the generation of circadian rhythms is a cellular process that involves interlocked autoregulatory transcriptional/translational feedback loops (Albrecht, 2002; Golombek et al., 2010; Kondratov et al., 2007). At the core loop, the positive elements CLOCK and BMAL1 form heterodimers and induce the transcription of the negative-feedback elements Period (Per1 and Per2) and Cryptochrome (Cry1 and Cry2), by binding to E-box elements at the promoter and enhancer regions of these genes. Their protein products, CRY and PER, form heterodimers that translocate to the nucleus where they inhibit the CLOCK/BMAL1 action by removing this complex from the E-box motifs of Cry and Per, and thus repress their own transcription (Fig. 1A). In an interlocking loop, the CLOCK/BMAL1 complex also activates the ROR (ROR $\alpha, R O R \beta$ and RORY) and REV-ERB (REV-ERB $\alpha$ and REVERB $\beta$ ) proteins. These proteins bind to ROR response elements (RORE) within the Bmal1 and Clock genes and activate or repress their expression respectively (Fig. 1B). The tight coordination of the positive and negative elements of transcription, as well as posttranscriptional and post-translational modifications, impose time delays that produce an accurate and robust cellular oscillator with a $24 \mathrm{~h}$ periodicity (Reppert et al., 2002). Cell autonomous clocks are ubiquitously expressed throughout the mammalian body (Yoo et al., 2004). The rhythm generated by these individual oscillators at the cellular level results in a coherent rhythmic output of the tissue that finally elicits a rhythmic behavior or physiological function. In that perspective, the incorporation of time information within the molecular clock serves one major purpose: to impose a temporal organization of key physiological processes. This is done through the circadian regulation of specific downstream genes, called clock-controlled genes (CCGs) that are involved in physiological functions such as metabolism and cell cycle (Panda et al., 2002b; Storch et al., 2002b). In fact, 10-20\% of mRNA transcripts are found to be regulated in a circadian fashion in various tissues (Akhtar et al., 2002; Hughes et al., 2009; Panda et al., 2002a; Storch et al., 2002a; Ueda et al., 2002). The transcription cycles of CCGS are controlled by core clock components such as BMAL1 (Rey et al., 2011), as well as by transcription factors of the bZIP family (DPB, TEF, HLF and E4BP4) whose rhythmic expression is governed by core clock proteins (Gachon et al., 2006; Mitsui et al., 2001). The mechanism of this rhythmic transcriptional control resembles that of the molecular clock: the binding of transcriptional factors to cis-regulatory elements presented on the promoter areas of CCGs in order to activate or repress their expression (Fig. 1C). 


\subsection{The circadian system: A symphony of rhythms}

127

Although the generation of circadian rhythms is a property of individual cells, the behavioral output requires the coordination of a complex clock network, which constitutes the circadian system. At the top of the system's hierarchy is the suprachiasmatic nucleus (SCN), a structure located in the hypothalamus. Classical lesion and transplantation studies (Moore et al., 1972; Ralph et al., 1990; Stephan et al., 1972) revealed that the SCN is both necessary and sufficient for the generation of behavioral rhythmicity, and was therefore named the master clock of the body. The SCN receives photic information from the retina, directly through retinohypothalamic fibers, in order to adjust its rhythms to environmental signals. This time-related information is then transmitted to other clocks in the brain, such as the olfactory bulbs and the hippocampus (Guilding et al., 2007) and clocks in the periphery, including liver, muscle and adrenal glands (Richards et al., 2012). The communication between the SCN and the peripheral clocks is mediated through the autonomic system and systemic cues such as body temperature, hormonal signaling and feeding (Challet, 2015; Mohawk et al., 2012) (Fig. 2). Finally, feedback information from peripheral clocks travel back to the SCN to provide a representation of the internal status of the body. Although the SCN functions as a master synchronizer of the circadian system, peripheral clocks are shown to be autonomous and self-sustained. This was clearly demonstrated in the study of Yoo et al. (Yoo et al., 2004) where peripheral clocks of SCNlesioned mice showed persistent circadian rhythms. However, these rhythms become desynchronized in the absence of SCN, meaning that they no longer tell the time correctly (Yoo et al., 2004). Overall, these findings support a hierarchical relationship between the SCN and the peripheral clocks as the one found in an "orchestra" - the SCN being the conductor and peripheral clocks being the instruments that follow the conductor's directions in order to provide a synchronous or "well-orchestrated" rhythmic circadian physiology.

\subsection{Circadian physiology: Why we need the peripheral clocks}

The role of the circadian system in mammalian physiology has been extensively studied in the past 15 years and it is now well-established that a large number of physiological functions are subjected to temporal regulation. Sleep and wake cycle, feeding behavior, metabolism, immune responses, blood pressure, urine production, detoxification and 
hormone release are a few overt examples (Gachon et al., 2004; Richards et al., 2013). In anticipation of the resting phase, core body temperature falls, anabolic processes increase and melatonin is secreted, whereas in anticipation of the active phase catabolism increases and glucocorticoids rise to prepare animals for the physiological demands of the day.

The discovery of peripheral clocks, initially in fibroblasts (Balsalobre et al., 1998) and later in a variety of organs (Yamazaki et al., 2000; Yoo et al., 2004), was of major importance for the circadian field because of their critical role in regulating circadian physiology. The ability of peripheral tissues to maintain cell-autonomous circadian oscillations, together with the hierarchical structure of the circadian system, suggests that circadian programming of physiology is to a large degree controlled by peripheral oscillators. In support of this notion, a study by Panda et al. compared the overlap between cyclic transcripts in the SCN and liver (total 650 transcripts) and found only 28 in common (Panda et al., 2002b). Therefore, the circadian regulation of the majority of output genes is tissue-specific and each peripheral clock is responsible for a specific output programme matched to the local needs. As discussed in the previous section (1.2), the temporal arrangement of tissue-specific functions is mediated through the expression of CCGs. Cis-regulatory elements, present throughout the genome, can activate or suppress the expression of CCGs. By a combination of these elements, the peak time of CCG expression is appropriately regulated, creating a wide distribution of cyclic activity within the tissue. Subsequently, this allows distinct biological pathways to operate at particular time windows. For instance, this is what allows the liver to separate two chemically antagonistic processes in time, such as gluconeogenesis and glycolysis.

The regulation of circadian physiology is to a large degree independent of the SCN. An example is a study showing that a targeted deletion of the clock in pancreatic islets results in reduced insulin secretion and leads to diabetes (Marcheva et al., 2010). Indirect control of tissue-specific physiology can be imposed by the SCN via endocrine signals (e.g. glucocorticoids), temperature and feeding cues. The circadian organization ensures tissue homeostasis and thus a temporal misalignment between the SCN and the peripheral clocks can jeopardize the optimal function of the various organs. This situation is reflected very well in the pathologies caused by jet lag, including immune dysfunction and metabolic syndrome (Brown et al., 2013). Finally, the peripheral clock-dependent circadian physiology will 
consequently lead to a circadian variation in disease susceptibility. In humans, cardiovascular episodes peak in the morning hours and are linked to the circadian fluctuations in blood pressure, heart rate and vasodilation (Casetta et al., 2002; Muller, 1999). Humans with polymorphisms in core clock genes (e.g. hPER2, hCRY1, hCLOCK, hBMAL1) display a great number of metabolic symptoms that highlight the translational relevance of the experimental findings (Dibner et al., 2015). Thus, a better understanding of the interplay between the circadian system and physiological systems is of great clinical importance.

\section{Circadian regulation of auditory function}

\subsection{Sensory regulation of circadian function}

Although the light-dark cycle is the primary zeitgeber of the circadian system, social cues through auditory and olfactory stimuli can serve as synchronizers of circadian rhythms. These social signals are aiming to synchronize the members of a social group, thus increasing their survival rate (Davidson et al., 2003). Auditory cues can act as strong zeitgebers in a variety of animals as well as humans. For instance, the behavioral rhythms of the house sparrow (Passer domesticus) can be entrained by conspecific bird songs (Menaker et al., 1966) and even by white noise (Reebs, 1989). Mice also show circadian responses to social cues, while other rodents like rats and hamsters do not (Davidson et al., 2003). The efficacy of auditory stimuli entrainment thus varies among different species. In humans, nocturnal presentation of an auditory stimulus can phase shift circadian rhythms of melatonin and core body temperature (Goel, 2005), as well as synchronize activity rhythms (Davidson et al., 2003). Taken together, these studies demonstrate that the auditory system is involved in circadian entrainment. However, little is known about circadian regulation in the auditory system. Earlier studies in rats has shown a circadian regulation of acoustic startle response (Chabot et al., 1992; Frankland et al., 1995) and a diurnal sensitivity to antibiotic- induced ototoxicity (Soulban et al., 1990; Yonovitz et al., 1991). Finally, in a 1958 study of audiogenic responses in mice, an increased susceptibility to convulsion was observed during the nocturnal phase (Halberg et al., 1958). Recently, our group revealed diurnal effects on noise sensitivity and otoprotection (Meltser et al., 2014) that we will discuss in the following paragraphs. 
220

The mammalian acoustic startle response (ASR) is a fast motor reflex elicited by a sudden, unexpected high-intensity acoustic stimulus that can provide information about an animal's hearing sensitivity (Heffner et al., 2006). The reflex involves activation of the cochlea root neurons and subsequently the motor neurons and skeletal muscles of the body (Lee et al., 1996). Circadian modulation of the ASR amplitude has been described in rats and was shown to be persistent under constant dark conditions, suggesting an endogenous mechanism (Chabot et al., 1992; Frankland et al., 1995). Recently, we found that mice also demonstrate diurnal ASR responses. Mice display greater startle responses (15\%) when exposed to a short acoustic stimulus of $120 \mathrm{~dB}$ SPL in the morning (ZT 3-6) in comparison to the evening (ZT 14-16) (Fig. 3A). In addition, the startle latency (time until maximal response) is greater in the evening than in the day (Meltser et al., 2014). Mice are nocturnal rodents, thus being more active during the night and at rest during the daytime. In this respect, these findings demonstrate that during the active phase, the startle reflex is slower with lower magnitude responses than during the resting phase. These results are consistent with the notion that motor activity reduces startle amplitude. However, in rats, this phenomenon is inversed: the amplitude of locomotor activity correlates with ASR amplitude (Frankland et al., 1995). These findings imply that locomotor activity and the startle reflex are probably independently modulated. In humans, the ASR differences are similar to those found in mice albeit inverted in time since humans are diurnal (Miller et al., 2006). More specifically, there is a $50 \%$ increase in startle amplitude in the evening, when cortisol levels are suppressed (Miller et al., 2006). In normal conditions, cortisol levels show a circadian pattern with a peak time in the morning followed by a drop in the evening. These finding are in line with reports demonstrating that pharmacological treatment with cortisol in the evening, when endogenous levels are low, reduces the startle response in humans (Buchanan et al., 2001; Liang et al., 1992).

\subsection{Diurnal sensitivity to noise trauma}

There are a variety of factors that can modulate auditory sensitivity to noise trauma such as genetic background (Lavinsky et al., 2015), stress (Canlon et al., 2007) and age (Li, 1992). We 
recently discovered that auditory sensitivity to noise trauma varies over the day. We found that challenging mice with a mild noise exposure at two different times of the day, in the morning (ZT3) and in the evening (ZT15), resulted in different degrees of hearing recovery. These two time points were selected because they correspond to the peak and trough levels of cochlear Per2 mRNA and protein expression as well as locomotor activity. CBA mice exposed to a noise trauma (6-12 kHz broadband noise of $100 \mathrm{~dB}$ SPL, for $1 \mathrm{~h}$ ) in the morning display complete recovery of their hearing thresholds after two weeks whereas those exposed in the evening still have 10-20 dB hearing threshold shifts, revealing a permanent damage (Fig. 3B). We previously described this noise paradigm to trigger a temporary threshold shift (TTS) with no loss of auditory hair cells when delivered during the day (Meltser et al., 2010). This unexpected permanent threshold shift (PTS) observed in the night noise exposed group indicates that the auditory system is more vulnerable at night. This report revealed that the capacity to recover from a noise trauma depends on the time exposure. It has been shown that diurnal sensitivity to noise is independent of behavior since animals displayed similar locomotor activity during day and night noise exposure (Park et al., 2016). Performing additional noise traumas at different time points throughout the day could demonstrate how the recovery of the auditory system to noise trauma behaves around the clock. Measures of wave I amplitude responses before and after noise trauma would give information about circadian variations in synaptic activity. Moreover, studies in humans have shown a 24-h variability of frequency for spontaneous optoacoustic emissions (SOAE) (Haggerty et al., 1993) and distortion product otoacoustic emissions (DPOAE) (Cacace et al., 1996), indicating a circadian influence on normal outer hair cell function. The inclusion of DPOAE threshold and input/output function would further characterize the circadian effects in the auditory system. Investigating the molecular pathways involved in the differential sensitivity to noise-induced hearing loss (NIHL) will increase our knowledge of the mechanisms involving resilience or vulnerability upon noise overexposure.

\section{The molecular clock in the cochlea}

\section{PER2::LUC oscillations in cochleae explants}

The transgenic PERIOD2::LUCIFEREASE (PER2::LUC) reporter mice (Yoo et al., 2004) allows real-time monitoring of gene expression rhythms. In these mice, the luciferase reporter (LUC) is fused in-frame to the C-terminal of the endogenous mPer2 locus, thus creating a 
mPER2::LUC fusion protein. This reporter system is very unique because it allows real-time luminescence monitoring of transcriptional and post-transcriptional regulation of the endogenous MPER2 protein.

Using PER2::LUC mice, we developed an organotypic culture that allowed real-time monitoring of PER2 dynamics in the cochlea (Meltser et al., 2014). These studies revealed that the isolated adult mouse cochlea is capable of generating robust, self-sustained PER2 oscillations that persist for at least one week, without medium exchange or other pharmacological manipulations. Interestingly, the rhythm amplitude of cochlear clock is higher than that of the liver clock, indicating that the cochlea possesses a very vigorous oscillator (Fig. 4) (Melster et. al., 2014). The period and phase of the cochlea clock is similar to that of the liver and the SCN (Melster et. al., 2014). PER2 is mainly located in the sensory cells of the organ of Corti (inner and outer hair cells) and in the spiral ganglion neurons (SGN) (Fig. 4), suggesting that the PER2 oscillations are likely to originate from these cell populations. These findings show that the cochlea contains a molecular clock which is capable of sustaining strong oscillations in culture. This is consistent with the study of Yoo et al. showing that some peripheral tissues, such as liver and pituitary gland, can generate selfsustained rather than damped circadian rhythms in the absence of rhythmic input, suggesting the existence of organ-specific synchronization mechanisms (Yoo et al., 2004). In contrast, some other peripheral oscillators (e.g kidney) seem to require entrainment signals or else their rhythms fade out rapidly in vitro (Yoo et al., 2004). This difference suggest that the requirements for sustained oscillations vary among different peripheral clocks, with some clocks having greater capacity for autonomous oscillations than others, as it is the case for the cochlea.

\section{Circadian gene expression rhythms in the cochlea}

The PER2::LUC real-time reporting of circadian dynamics is a useful method for studying the function of the molecular clock. However, the PER2 is only one of many core clock proteins that contribute to the generation of circadian oscillations. As described earlier, the molecular clock machinery involves genes that act as transcriptional activators or repressors and they are common in all clock systems of the body. In spite of the universal presence of these clock genes, their expression levels may vary between different tissues, and each 
tissue may also have complementary clock genes that can specifically modulate clock output genes. Subtle variations in clock gene expression patterns may be very important for tissuespecific functions. It is not surprising that $10 \%$ of a given transcriptome is rhythmic, but the overlap of rhythmic transcripts between 2 tissues may be not more than $20 \%$ (Panda et al., 2002b). Thus, understanding the tissue-specific circadian networks is important for unravelling the mechanisms of circadian physiology in a given organ.

In the cochlea, we revealed by quantitative real-time polymerase chain reaction (qRT-PCR) circadian regulation of a variety of clock mRNA transcripts including Per1, Per2, Bmal1, Clock, and Rev-Erba (Meltser et al., 2014). These results confirmed the reversed phase correlation between the positive (Bmal1 and Clock) and the negative (Per1 and Per2) elements of the molecular clock. In addition, using the Nanostring nCounter technology (Geiss et al., 2008; Vikhe Patil et al., 2015), which is an ultrasensitive method to measure mRNA transcript abundance, we identified the circadian expression of the transcription factors Dbp, HIF, and Tef (unpublished observations). They belong to the PAR-B-ZIP (proline- and acidic amino acid-rich basic leucine zipper) family and they are controlled by the CLOCK and BMAL1 proteins that bind to E-box elements within these genes (Ripperger et al., 2000). In liver, they are known to regulate the circadian expression of CCGs involved in metabolism, through binding to D-box motifs of downstream targets (Gachon et al., 2006). Therefore, many CCGs are indirectly controlled by the molecular clock through rhythmically expressed transcription factors. In the cochlea, $D p b$ is shown to be downregulated after noise trauma (Gratton et al., 2011), and could thus be an important regulator of the circadian cochlear response to noise. Furthermore, we examined the temporal expression patterns of several early-response genes involved in cochlear function and found rhythmic transcript levels of Jun-B and Zif268 (unpublished observations). They are involved in synaptic plasticity and induction of long term potentiation (LTP) (Cole et al., 1989; Wisden et al., 1990). In birds, zenk (the homologue of zif268) is upregulated in auditory regions in response to novel songbird learning (Gentner et al., 2004) and an upregulation of Jun-b occurs in the mouse cochlea after noise exposure (Gratton et al., 2011). Whether clock-controlled genes determine noise sensitivity at different times of the day remains to be uncovered. 
In mice, recovery of ABR thresholds is dependent on the time of noise exposure, with more damage occurring after being delivered at night. There is a clear correlation between noise sensitivity throughout the day and the presence of a circadian clock in the cochlea. Although the role of the clock machinery on noise sensitivity remains to be established (e.g. by knocking down core clock genes), we found that noise alters the cochlear clock responses in mRNA and protein (Meltser et al., 2014). For example, cochlear explants from nightstimulated mice, cultured for a period of 6 days, show a $27 \%$ decrease in PER2::LUC rhythm amplitude compared to the sham group (Fig. 5A). Similarly, the amplitude of Per2 mRNA transcripts decreases by $30 \%$ in cochleae from night exposed mice (Fig. 5B). Moreover, amplitude suppression is found in Per1 and Rev-Erb $\alpha$ mRNA transcripts. Finally, the amplitude of Bmal1 increases following night exposure, suggesting reduced negative feedback from Rev-Erbo (Fig. 5B). Together, these findings show that the increased sensitivity to damage after night noise is correlated with a suppression of circadian transcript profiles in the cochlea.

The next step in understanding the circadian regulation of noise sensitivity would be the identification of clock output genes that are involved in susceptibility/protection against noise damage and those involved in recovery processes. Due to the amount of potential circadian target genes, transcriptome analysis of the cochlea after day or night noise injury would be needed to reveal the whole circadian network in the cochlea. However, by narrowing down possible mechanisms of auditory damage, we were able to identify a strong candidate that links the cochlear clock to the recovery of synaptic function, following a nocturnal noise exposure. Permanent auditory damage in absence of hair cell loss could derive from several mechanisms such as damage at the level of the stereocilia, damage at the level of outer hair cell's electromotile abilities, damage at the inner hair cells-afferent neuron synapse (Kujawa et al., 2015). We privileged the latter since neurotrophic factors such as the brain derived neurotrophic factor (BDNF) are known to display a circadian expression pattern in the brain (Liang et al., 1998) and BDNF has a longstanding importance in cochlear neurogenesis and synaptic homeostasis (Singer et al., 2014). In the following 
paragraph, we will discuss how the circadian control of BDNF has emerged as a circadian repair mechanism after noise damage.

\section{Circadian regulation of BDNF activation mediates otoprotection}

Using the NanoString nCounter technology we found peak mRNA expression for the $B d n f$ variants 1 and 4 at ZT 16 (unpublished observations). We next measured the transcriptional response to day or night noise trauma and found a striking 35 fold change in $B d n f$ mRNA transcript levels after day noise exposure, whereas night noise exposure only triggered a 2fold increase. We thus hypothesized that the ability of the hearing system to recover from day noise trauma was linked to the capacity of the cochlea to produce more Bdnf in response noise. BDNF and neurotrophin-3 (NT-3) are two major neurotrophins involved in cochlear neuro- and synapto-genesis (Ramekers et al., 2012). NT-3 null mice display severe spiral ganglion neuronal loss (84\%) whereas BDNF null mice mainly lose vestibular neurons (Fritzsch et al., 2004). In the cochlea, loss of BDNF function yields a loss of high-frequency neurons and as a consequence, the function of NT-3 appears to be predominant over that of BDNF regarding auditory neuron survival. However, genetic studies in mice have convincingly shown that BDNF overexpression could restore auditory neuronal growth in NT3 mutant mice (Fritzsch et al., 2004). BDNF and NT-3 receptors, TrkB and TrkC respectively, are expressed in auditory neurons. Quite similar to the phenotype of the NT-3 and BDNF mutants, loss of TrkC function is much more severe than loss of TrkB function resulting in a loss of $50 \%$ of auditory neurons. However, knock-out of TrkB in a TrkC -/- background causes a complete absence of auditory neurons. Overall, these studies highlight the importance of BDNF/TrkB signaling in cochlear neurogenesis (Fritzsch et al., 2004) . In vitro studies in the post-natal cochlea have shown that both neurotrophins trigger the regeneration of synapses after excitotoxic injury (Wang et al., 2011).

Pharmacological screenings have identified a powerful and selective agonist of the TrkB receptor, namely 7,8 dihydroxyflavone (DHF) (Jang et al., 2010), which is a potential alternative to BDNF that has a very short life-span. DHF has been found to be a potent ligand of TrkB, activating TrkB signaling in neurons but not in those lacking a functional TrkB. DHF also prevents glutamate-mediated apoptosis (Jang et al., 2010). In the cochlea, DHF has been shown to promote auditory protection in mice lacking connexin 26 (Yu et al., 2013), which 
403

404

405

406

407

408

409

410

411

412

413

414

415

416

417

418

419

420

421

422

423

424

425

426

427

428

indicates that DHF can cross the ear-blood barrier and target cochlear TrkB. We tested whether DHF would be able to protect auditory function from night noise exposure. We found that systemic administration of DHF prior to night noise exposure prevents from permanent damage and that hearing thresholds recover to normal levels (Fig. 6). At the synaptic level, DHF can rescue, albeit not completely, from the loss of presynaptic ribbons commonly found after moderate noise overexposure (Meltser et al., 2014).

\section{Conclusions}

The discovery of a clock machinery in the cochlea is of critical importance since it opens the path towards a completely new area of research likely applicable to most aspects of auditory physiology. It remains to be determined if alterations in circadian rhythms increase vulnerability to a broad spectrum of auditory insults other than noise trauma shown here (e.g. ototoxic drugs, ischemia). These findings are undoubtedly of major relevance for humans, due to the well-established relationship between experimental and clinical work on circadian rhythms. The emerging importance of hidden hearing loss, accompanied by the evidence that de-afferentation in the cochlea is an early indicator of auditory alterations, stresses the need for investigating auditory functions in humans with circadian disruptions such as shift workers, or flight crew operating in long-distance longitudinal flights. Finally, what are the environmental cues that modulate the cochlear clock? What are the molecular components of the clock machinery that drive vulnerability or resilience to noise? It would also be valuable to know if these effects are auditory-specific, or whether they involve system-wide changes. Addressing these important questions will provide new avenues for understanding the mechanisms underlying auditory damage and optimize preventive and therapeutic interventions adapted in time. 
Akhtar, R.A., Reddy, A.B., Maywood, E.S., Clayton, J.D., King, V.M., Smith, A.G., Gant, T.W., Hastings, M.H., Kyriacou, C.P., 2002. Circadian cycling of the mouse liver transcriptome, as revealed by cDNA microarray, is driven by the suprachiasmatic nucleus. Curr Biol 12, 540-50.

Albrecht, U., 2002. Invited Review: Regulation of mammalian circadian clock genes. Journal of Applied Physiology 92, 1348-1355.

Balsalobre, A., Damiola, F., Schibler, U., 1998. A serum shock induces circadian gene expression in mammalian tissue culture cells. Cell 93, 929-37.

Brown, S., Azzi, A. 2013. Peripheral Circadian Oscillators in Mammals. In: Kramer, A., Merrow, M., (Eds.), Circadian Clocks, Vol. 217. Springer Berlin Heidelberg. pp. 45-66.

Buchanan, T.W., Brechtel, A., Sollers, J.J., Lovallo, W.R., 2001. Exogenous cortisol exerts effects on the startle reflex independent of emotional modulation. Pharmacol Biochem Behav 68, 20310.

Cacace, A.T., McClelland, W.A., Weiner, J., McFarland, D.J., 1996. Individual differences and the reliability of 2F1-F2 distortion-product otoacoustic emissions: effects of time-of-day, stimulus variables, and gender. J Speech Hear Res 39, 1138-48.

Canlon, B., Meltser, I., Johansson, P., Tahera, Y., 2007. Glucocorticoid receptors modulate auditory sensitivity to acoustic trauma. Hear Res 226, 61-9.

Casetta, I., Granieri, E., Portaluppi, F., Manfredini, R., 2002. CIrcadian variability in hemorrhagic stroke. JAMA 287, 1266-1267.

Chabot, C.C., Taylor, D.H., 1992. Circadian modulation of the rat acoustic startle response. Behavioral Neuroscience 106, 846-852.

Challet, E., 2015. Keeping circadian time with hormones. Diabetes, Obesity and Metabolism 17, 76 83.

Cole, A.J., Saffen, D.W., Baraban, J.M., Worley, P.F., 1989. Rapid increase of an immediate early gene messenger RNA in hippocampal neurons by synaptic NMDA receptor activation. Nature 340, 474-6.

Davidson, A.J., Menaker, M., 2003. Birds of a feather clock together--sometimes: social synchronization of circadian rhythms. Curr Opin Neurobiol 13, 765-9.

Dibner, C., Schibler, U., 2015. Circadian timing of metabolism in animal models and humans. J Intern Med 277, 513-27.

Frankland, P.W., Ralph, M.R., 1995. Circadian modulation in the rat acoustic startle circuit. Behav Neurosci 109, 43-8.

Fritzsch, B., Tessarollo, L., Coppola, E., Reichardt, L.F., 2004. Neurotrophins in the ear: their roles in sensory neuron survival and fiber guidance. Prog Brain Res 146, 265-78.

Gachon, F., Nagoshi, E., Brown, S.A., Ripperger, J., Schibler, U., 2004. The mammalian circadian timing system: from gene expression to physiology. Chromosoma 113, 103-12.

Gachon, F., Olela, F.F., Schaad, O., Descombes, P., Schibler, U., 2006. The circadian PAR-domain basic leucine zipper transcription factors DBP, TEF, and HLF modulate basal and inducible xenobiotic detoxification. Cell Metab 4, 25-36.

Geiss, G.K., Bumgarner, R.E., Birditt, B., Dahl, T., Dowidar, N., Dunaway, D.L., Fell, H.P., Ferree, S., George, R.D., Grogan, T., James, J.J., Maysuria, M., Mitton, J.D., Oliveri, P., Osborn, J.L., Peng, T., Ratcliffe, A.L., Webster, P.J., Davidson, E.H., Hood, L., Dimitrov, K., 2008. Direct multiplexed measurement of gene expression with color-coded probe pairs. Nat Biotechnol 26, 317-25.

Gentner, T., Hulse, S., Ball, G., 2004. Functional differences in forebrain auditory regions during learned vocal recognition in songbirds. Journal of Comparative Physiology A 190, 1001-1010.

Goel, N., 2005. Late-night presentation of an auditory stimulus phase delays human circadian rhythms. Am J Physiol Regul Integr Comp Physiol 289, R209-16.

Golombek, D.A., Rosenstein, R.E. 2010. Physiology of Circadian Entrainment. 
Gratton, M.A., Eleftheriadou, A., Garcia, J., Verduzco, E., Martin, G.K., Lonsbury-Martin, B.L., Vazquez, A.E., 2011. Noise-induced changes in gene expression in the cochleae of mice differing in their susceptibility to noise damage. Hear Res 277, 211-26.

Guilding, C., Piggins, H.D., 2007. Challenging the omnipotence of the suprachiasmatic timekeeper: are circadian oscillators present throughout the mammalian brain? Eur J Neurosci 25, 3195216.

Haggerty, H.S., Lusted, H.S., Morton, S.C., 1993. Statistical quantification of 24-hour and monthly variabilities of spontaneous otoacoustic emission frequency in humans. Hear Res 70, 31-49.

Halberg, F., ELWOOD, JACOBSEN, WADSWORTH, G., BITTNER, J.J., 1958. Audiogenic Abnormality Spectra, Twenty-four Hour Periodicity, and Lighting. Science 128, 657-658.

Hardin, P.E., Hall, J.C., Rosbash, M., 1990. Feedback of the Drosophila period gene product on circadian cycling of its messenger RNA levels. Nature 343, 536-40.

Heffner, H.E., Koay, G., Heffner, R.S., 2006. Behavioral assessment of hearing in mice--conditioned suppression. Curr Protoc Neurosci Chapter 8, Unit8.21D.

Hughes, M.E., DiTacchio, L., Hayes, K.R., Vollmers, C., Pulivarthy, S., Baggs, J.E., Panda, S., Hogenesch, J.B., 2009. Harmonics of circadian gene transcription in mammals. PLoS Genet 5, e1000442.

Jang, S.W., Liu, X., Yepes, M., Shepherd, K.R., Miller, G.W., Liu, Y., Wilson, W.D., Xiao, G., Blanchi, B., Sun, Y.E., Ye, K., 2010. A selective TrkB agonist with potent neurotrophic activities by 7,8dihydroxyflavone. Proc Natl Acad Sci U S A 107, 2687-92.

Kondratov, R.V., Gorbacheva, V.Y., Antoch, M.P. 2007. The Role of Mammalian Circadian Proteins in Normal Physiology and Genotoxic Stress Responses. In: Gerald, P.S., (Ed.), Current Topics in Developmental Biology, Vol. Volume 78. Academic Press. pp. 173-216.

Konopka, R.J., Benzer, S., 1971. Clock Mutants of Drosophila melanogaster. Proceedings of the National Academy of Sciences 68, 2112-2116.

Kujawa, S.G., Liberman, M.C., 2015. Synaptopathy in the noise-exposed and aging cochlea: Primary neural degeneration in acquired sensorineural hearing loss. Hear Res 330, 191-9.

Lavinsky, J., Crow, A.L., Pan, C., Wang, J., Aaron, K.A., Ho, M.K., Li, Q., Salehide, P., Myint, A., MongesHernadez, M., Eskin, E., Allayee, H., Lusis, A.J., Friedman, R.A., 2015. Genome-wide association study identifies nox3 as a critical gene for susceptibility to noise-induced hearing loss. PLoS Genet 11, e1005094.

Lee, Y., Lopez, D.E., Meloni, E.G., Davis, M., 1996. A primary acoustic startle pathway: obligatory role of cochlear root neurons and the nucleus reticularis pontis caudalis. J Neurosci 16, 3775-89.

Li, H.S., 1992. Genetic influences on susceptibility of the auditory system to aging and environmental factors. Scand Audiol Suppl 36, 1-39.

Liang, F.Q., Walline, R., Earnest, D.J., 1998. Circadian rhythm of brain-derived neurotrophic factor in the rat suprachiasmatic nucleus. Neurosci Lett 242, 89-92.

Liang, K.C., Melia, K.R., Miserendino, M.J., Falls, W.A., Campeau, S., Davis, M., 1992. Corticotropinreleasing factor: long-lasting facilitation of the acoustic startle reflex. J Neurosci 12, 2303-12.

Marcheva, B., Ramsey, K.M., Buhr, E.D., Kobayashi, Y., Su, H., Ko, C.H., Ivanova, G., Omura, C., Mo, S., Vitaterna, M.H., Lopez, J.P., Philipson, L.H., Bradfield, C.A., Crosby, S.D., JeBailey, L., Wang, X., Takahashi, J.S., Bass, J., 2010. Disruption of the clock components CLOCK and BMAL1 leads to hypoinsulinaemia and diabetes. Nature 466, 627-31.

Meltser, I., Tahera, Y., Canlon, B., 2010. Differential activation of mitogen-activated protein kinases and brain-derived neurotrophic factor after temporary or permanent damage to a sensory system. Neuroscience 165, 1439-46.

Meltser, I., Cederroth, C.R., Basinou, V., Savelyev, S., Lundkvist, G.S., Canlon, B., 2014. TrkB-mediated protection against circadian sensitivity to noise trauma in the murine cochlea. Curr Biol 24, 658-63.

Menaker, M., Eskin, A., 1966. Entrainment of circadian rhythms by sound in Passer domesticus. Science 154, 1579-81.

Miller, M.W., Gronfier, C., 2006. Diurnal variation of the startle reflex in relation to HPA-axis activity in humans. Psychophysiology 43, 297-301. 
Mitsui, S., Yamaguchi, S., Matsuo, T., Ishida, Y., Okamura, H., 2001. Antagonistic role of E4BP4 and PAR proteins in the circadian oscillatory mechanism. Genes Dev 15, 995-1006.

Mohawk, J.A., Green, C.B., Takahashi, J.S., 2012. Central and peripheral circadian clocks in mammals. Annu Rev Neurosci 35, 445-62.

Moore, R.Y., Eichler, V.B., 1972. Loss of a circadian adrenal corticosterone rhythm following suprachiasmatic lesions in the rat. Brain Res 42, 201-6.

Muller, J.E., 1999. Circadian variation in cardiovascular events. American Journal of Hypertension 12 , 35S-42S.

Panda, S., Antoch, M.P., Miller, B.H., Su, A.I., Schook, A.B., Straume, M., Schultz, P.G., Kay, S.A., Takahashi, J.S., Hogenesch, J.B., 2002a. Coordinated Transcription of Key Pathways in the Mouse by the Circadian Clock. Cell 109, 307-320.

Panda, S., Antoch, M.P., Miller, B.H., Su, A.I., Schook, A.B., Straume, M., Schultz, P.G., Kay, S.A., Takahashi, J.S., Hogenesch, J.B., 2002b. Coordinated transcription of key pathways in the mouse by the circadian clock. Cell 109, 307-20.

Park, J.S., Cederroth, C.R., 2016. Identification of a Circadian Clock in the Inferior Colliculus and Its Dysregulation by Noise Exposure 36, 5509-19.

Ralph, M.R., Foster, R.G., Davis, F.C., Menaker, M., 1990. Transplanted suprachiasmatic nucleus determines circadian period. Science 247, 975-8.

Ramekers, D., Versnel, H., Grolman, W., Klis, S.F., 2012. Neurotrophins and their role in the cochlea. Hear Res 288, 19-33.

Reebs, S.G., 1989. Acoustical Entrainment of Circadian Activity Rhythms in House Sparrows: Constant Light is not Necessary. Ethology 80, 172-181.

Reppert, S.M., Weaver, D.R., 2002. Coordination of circadian timing in mammals. Nature 418, 935941.

Rey, G., Cesbron, F., Rougemont, J., Reinke, H., Brunner, M., Naef, F., 2011. Genome-wide and phasespecific DNA-binding rhythms of BMAL1 control circadian output functions in mouse liver. PLoS Biol 9, e1000595.

Richards, J., Gumz, M.L., 2012. Advances in understanding the peripheral circadian clocks. Faseb j 26, 3602-13.

Richards, J., Gumz, M.L., 2013. Mechanism of the circadian clock in physiology. Am J Physiol Regul Integr Comp Physiol 304, R1053-64.

Ripperger, J.A., Shearman, L.P., Reppert, S.M., Schibler, U., 2000. CLOCK, an essential pacemaker component, controls expression of the circadian transcription factor DBP. Genes Dev 14, 67989.

Singer, W., Panford-Walsh, R., Knipper, M., 2014. The function of BDNF in the adult auditory system. Neuropharmacology 76 Pt C, 719-28.

Soulban, G., Smolensky, M.H., Yonovitz, A., 1990. Gentamicin-Induced Chronotoxicity: Use of Body Temperature as a Orcadian Marker Rhythm. Chronobiology International 7, 393-402.

Stephan, F.K., Zucker, I., 1972. Circadian rhythms in drinking behavior and locomotor activity of rats are eliminated by hypothalamic lesions. Proc Natl Acad Sci U S A 69, 1583-6.

Storch, K.-F., Lipan, O., Leykin, I., Viswanathan, N., Davis, F.C., Wong, W.H., Weitz, C.J., $2002 a$. Extensive and divergent circadian gene expression in liver and heart. Nature 417, 78-83.

Storch, K.F., Lipan, O., Leykin, I., Viswanathan, N., Davis, F.C., Wong, W.H., Weitz, C.J., 2002 b. Extensive and divergent circadian gene expression in liver and heart. Nature 417, 78-83.

Ueda, H.R., Chen, W., Adachi, A., Wakamatsu, H., Hayashi, S., Takasugi, T., Nagano, M., Nakahama, K., Suzuki, Y., Sugano, S., lino, M., Shigeyoshi, Y., Hashimoto, S., 2002. A transcription factor response element for gene expression during circadian night. Nature 418, 534-9.

Vikhe Patil, K., Canlon, B., Cederroth, C.R., 2015. High quality RNA extraction of the mammalian cochlea for qRT-PCR and transcriptome analyses. Hear Res 325, 42-8.

Wang, Q., Green, S.H., 2011. Functional role of neurotrophin-3 in synapse regeneration by spiral ganglion neurons on inner hair cells after excitotoxic trauma in vitro. J Neurosci 31, 7938-49. 
Wisden, W., Errington, M.L., Williams, S., Dunnett, S.B., Waters, C., Hitchcock, D., Evan, G., Bliss, T.V., Hunt, S.P., 1990. Differential expression of immediate early genes in the hippocampus and spinal cord. Neuron 4, 603-14.

Yamazaki, S., Numano, R., Abe, M., Hida, A., Takahashi, R., Ueda, M., Block, G.D., Sakaki, Y., Menaker, M., Tei, H., 2000. Resetting central and peripheral circadian oscillators in transgenic rats. Science 288, 682-5.

Yonovitz, A., Fisch, J.E., 1991. Circadian Rhythm Dependent Kanamycin-induced Hearing Loss in Rodents Assessed by Auditory Brainstem Responses. Acta Oto-laryngologica 111, 1006-1012.

Yoo, S.H., Yamazaki, S., Lowrey, P.L., Shimomura, K., Ko, C.H., Buhr, E.D., Siepka, S.M., Hong, H.K., Oh, W.J., Yoo, O.J., Menaker, M., Takahashi, J.S., 2004. PERIOD2::LUCIFERASE real-time reporting of circadian dynamics reveals persistent circadian oscillations in mouse peripheral tissues. Proc Natl Acad Sci U S A 101, 5339-46.

Yu, Q., Chang, Q., Liu, X., Wang, Y., Li, H., Gong, S., Ye, K., Lin, X., 2013. Protection of spiral ganglion neurons from degeneration using small-molecule TrkB receptor agonists. J Neurosci 33, 13042-52.

\section{Figure Legends}

Fig. 1: The molecular clock machinery. A) Core loop: CLOCK (C) and BMAL1 (B) bind to E-box DNA motifs and induce the transcription of Per and Cry genes (Per1, Per2, Cry1 and Cry2). Accumulation of PER/CRY complexes inhibit the transactivation potential of $C$ and $B$, thereby repressing their own transcription. As a consequence, PER and CRY levels decline, allowing $C$ and $\mathrm{B}$ to initiate a new cycle of gene expression. Per1 and Per2 transcription can be additionally induced by systemically regulated transcriptional factors: Heat shock factor (HSF) binding to heat shock elements (HSE), CAMP responsive element (CRE)-binding protein (CREB) and glucocorticoid receptor (GR) binding to glucocorticoid responsive elements (GRE). B) Interlocking loop: CLOCK and BMAL1 activate the orphan nuclear receptor genes Ror (Rora, RorB and Rory) and Rev-Erb (Rev-Erba and Rev-ErbB). The transcription of Bmal1 and Clock is then regulated through competition between REV-ERB repressors and ROR activators, acting on retinoid-related orphan receptor response elements (RORE). C) Regulation of clock-controlled genes (CCGs): CLOCK and BMAL1 can regulate the transcription of CCGs by binding to E-box elements on their promoter area. These genes are then translated into CCG protein products and regulate physiological processes in a temporal way.

Fig. 2: Organization of the circadian system. The suprachiasmatic nucleus (SCN) is the major pacemaker of the circadian system that receives photic information directly from the retina and synchronizes peripheral oscillators found in other brain areas and peripheral 
tissues (entrainment). This is mediated through autonomic innervation, humoral signals, hormones and the regulation of body temperature and feeding.

Fig. 3. Diurnal variation of auditory function. A) Acoustic startle response (ASR) in CBA mice is greater in the morning (ZT 3-6, red) compared to night (ZT 14-16, blue). B) Auditory brainstem response ( $A B R$ ) of CBA mice exposed to noise in the morning (ZT 3-5, red) or at night (ZT 14-16, blue) show similar levels of hearing damage when measured $24 \mathrm{~h}$ post exposure. However, 2 weeks later the morning group shows complete recovery of their threshold shifts, whereas the night group still shows high threshold shifts, thereby permanent hearing damage. Modified with permission from Cell Press (Melster et. al., 2014).

Fig. 4. Circadian PER2::LUC rhythms in the adult cochlea. Representative bioluminescence recordings of circadian PER2::LUC expression from cultured cochlea, liver and SCN explants. In the adult cochlea PER2 is mainly expressed in inner hair cells, outer hair cells and in the spiral ganglion neurons. Modified with permission from Cell Press (Melster et. al., 2014).

Fig. 5. Effects of noise exposure on the cochlear clock. A) Cochlear PER2::LUC rhythm amplitude after exposure to a morning (ZT 3-5, red) or a night (ZT14-16, blue) noise trauma. Night noise significantly reduces the rhythm amplitude of the PER2::LUC oscillation, whereas day noise does not. B) Temporal expression of Per2, Per1, Rev-Erbo, and Bmal1 mRNAs in cochlea from non-exposed mice (white circles) or exposed to a morning (ZT 3, red circles) or night (ZT 15, blue circles) noise trauma. Night noise supresses the amplitude of Per2, Per1 and Rev-Erb $\alpha$ rhythms and increases the amplitude of Bmal1 rhythms. Modified with permission from Cell Press (Melster et. al., 2014).

Fig. 6. Regulation of diurnal noise sensitivity by Bdnf. A) Bdnf mRNA expression in the cochlea after a noise exposure in the morning (ZT 3-5, red) or at night (ZT 14-16, blue). Day noise trauma increases $B d n f$ mRNA by 35 -fold. B) ABR threshold shifts from mice exposed to noise in the morning (ZT 3-5, red) or at night (ZT 14-16, blue), measured $24 \mathrm{hr}$ post (left panel) and 2 weeks post (right panel). DMSO-treated animals (white circles) and DHF-treated animals (filled circles). DHF administration prevents from permanent hearing loss in the night exposed group, as hearing thresholds return to normal levels 2 weeks after noise exposure. The lack of $B d n f$ induction after night noise and the hearing threshold rescue by 
647 DHF suggest that BDNF signalling contributes to the diurnal sensitivity to noise damage.

648 Modified with permission from Cell Press (Melster et. al., 2014). 


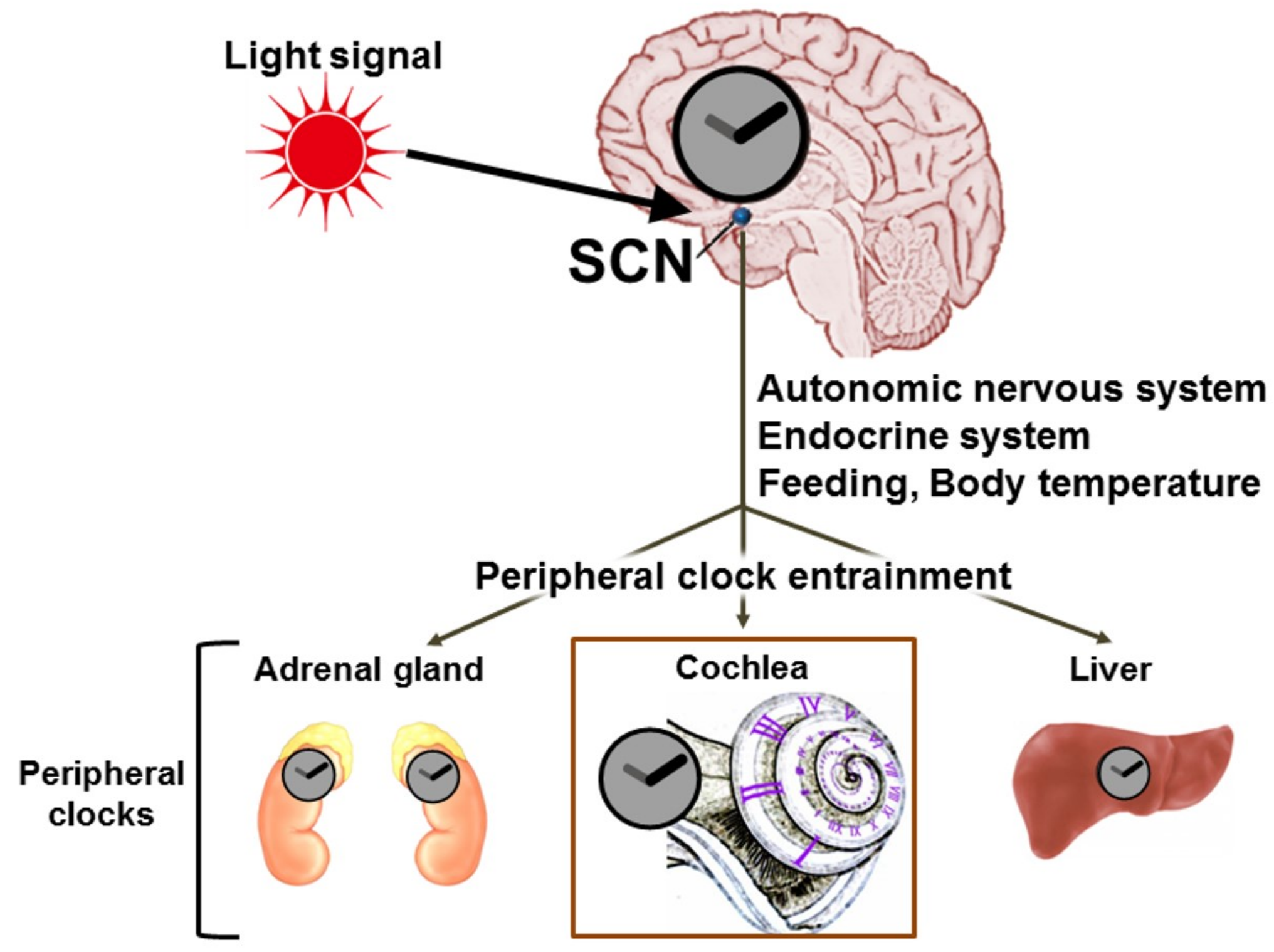


A

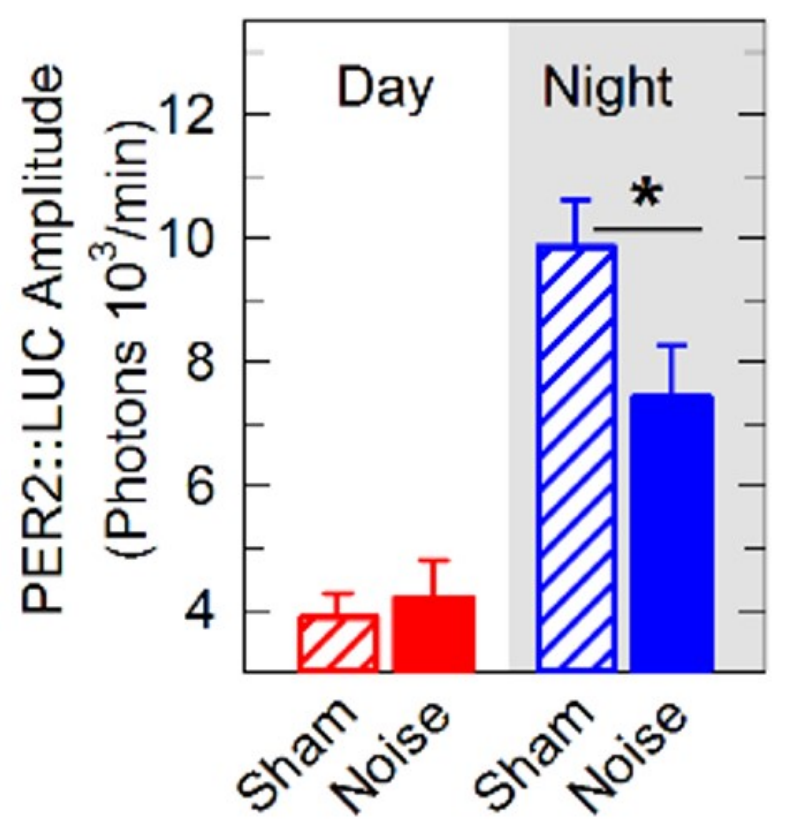

B

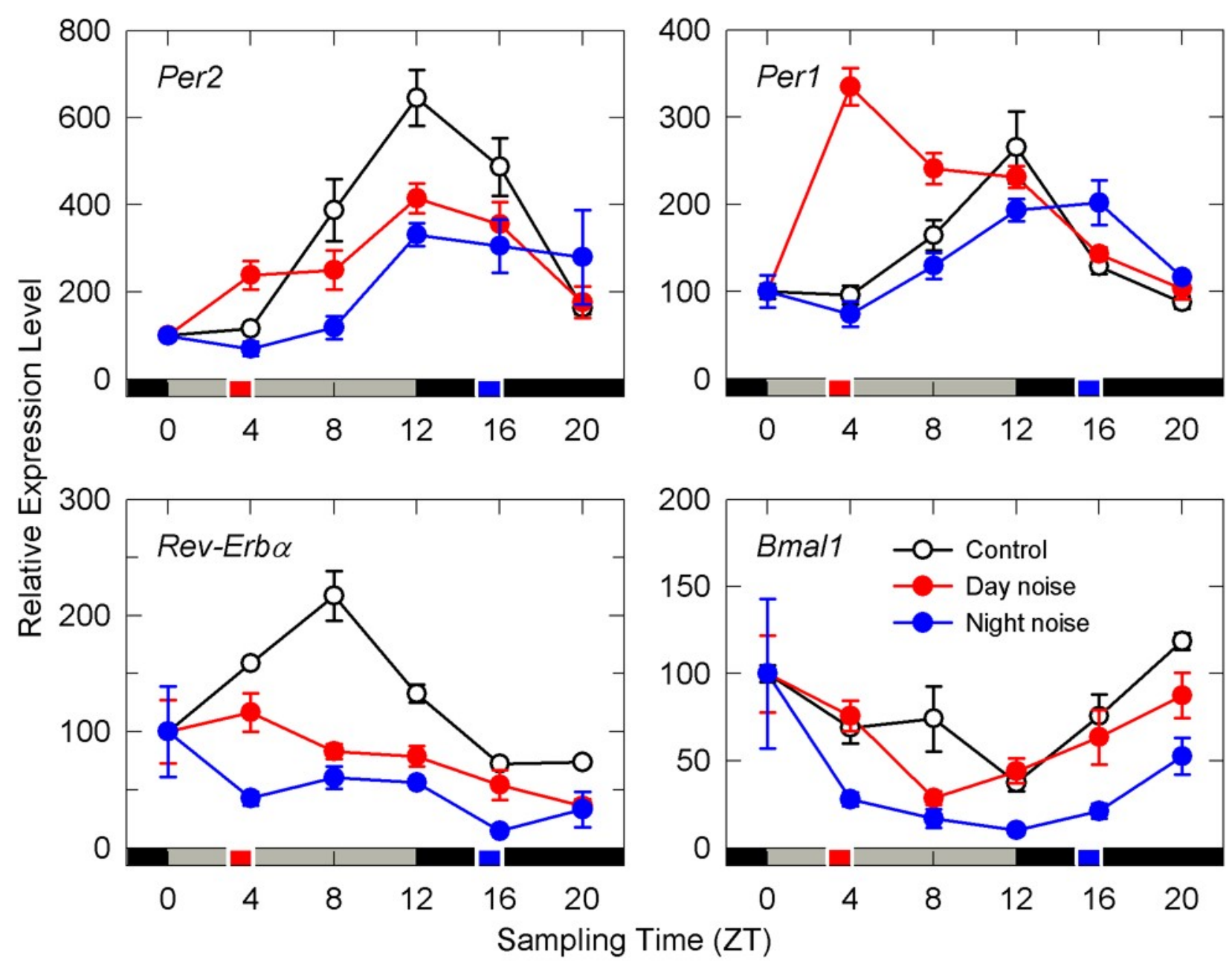



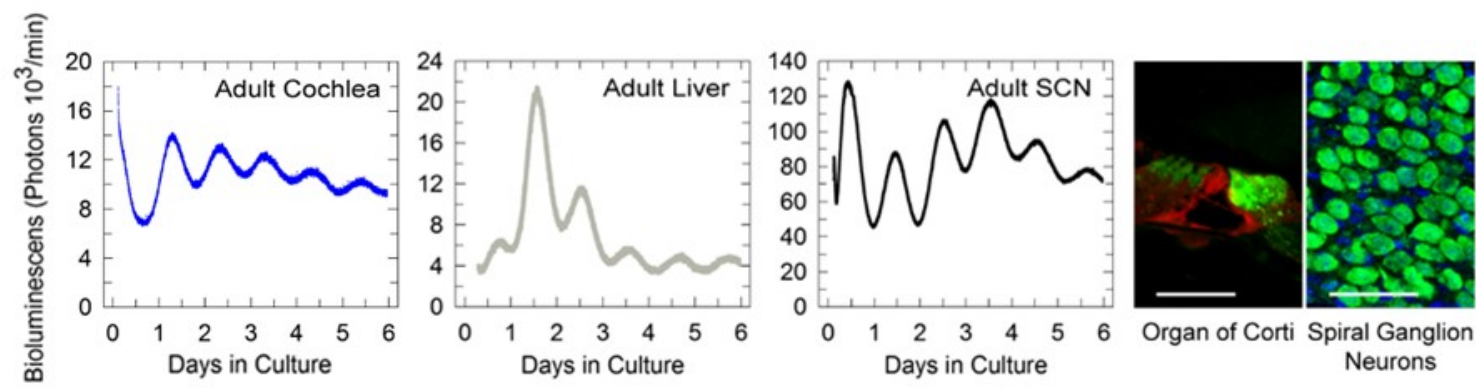

Organ of Corti Spiral Ganglion Neurons 


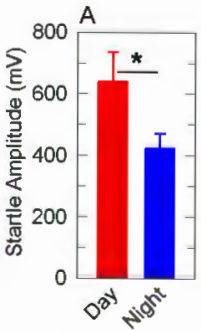

B

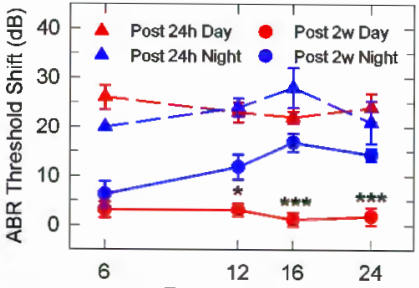

Frequency $(\mathrm{kHz})$ 

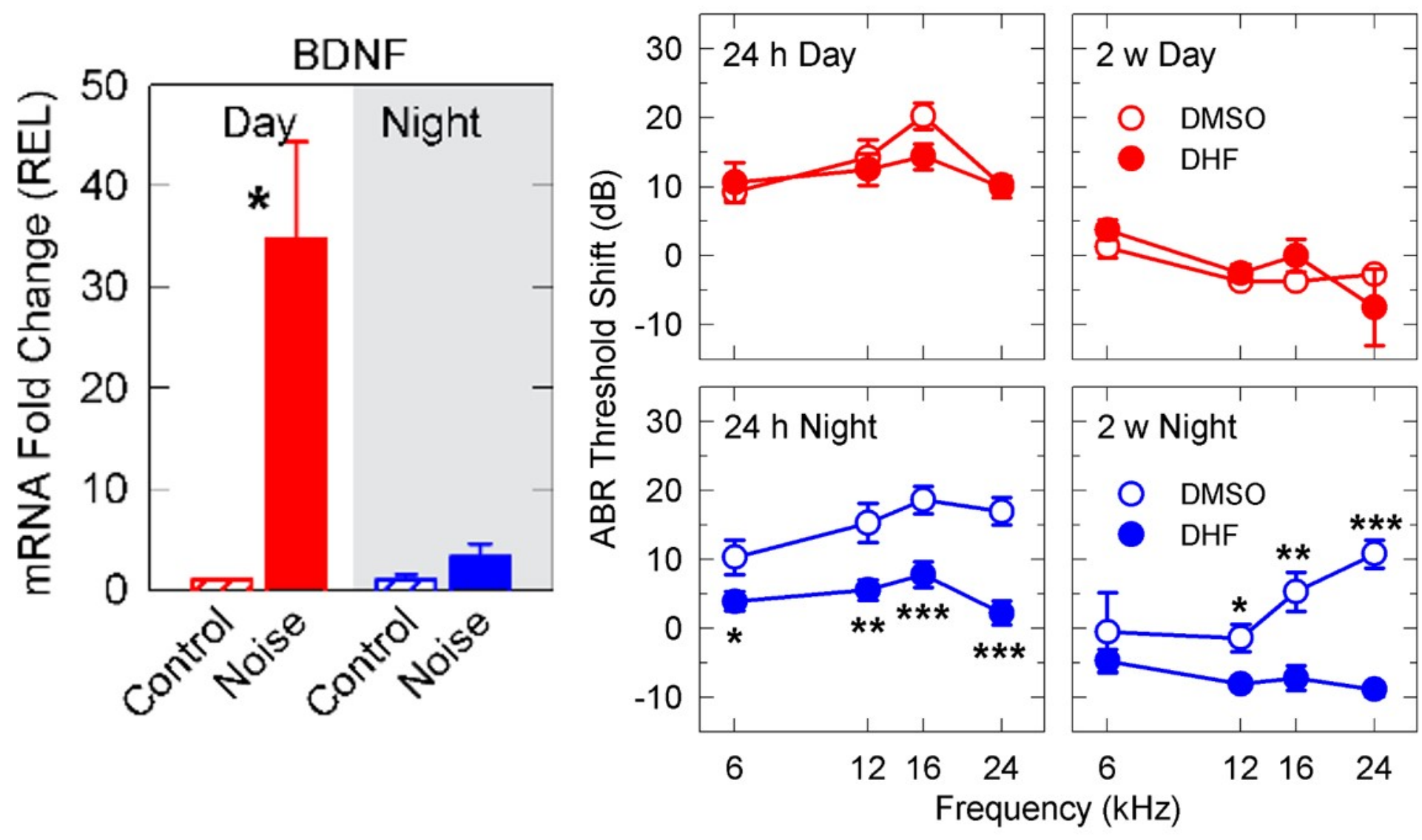
Post Noise Trauma

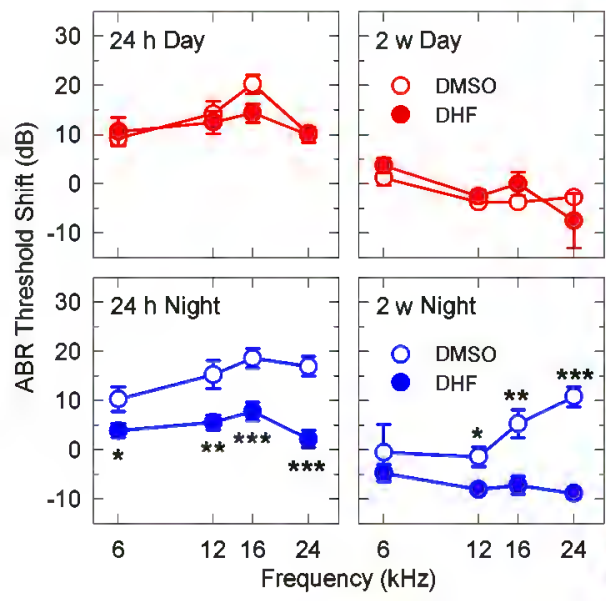

Distance from Apex

$3.5 \mathrm{~mm}$

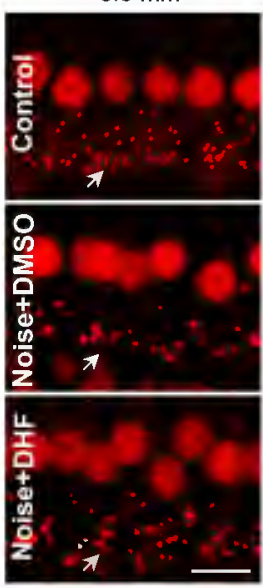

Frequency $(\mathrm{kHz})$

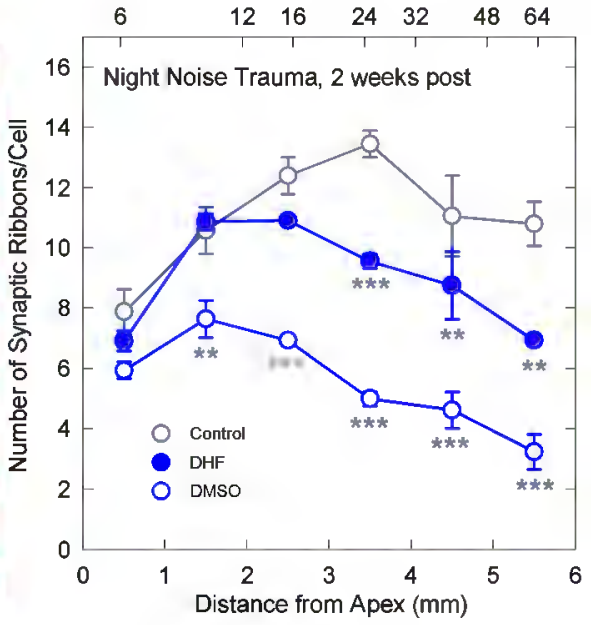

